\title{
Factors associated with prolonged hospitalization, readmission, and death in elderly heart failure patients in western Romania
}

This article was published in the following Dove Press journal:

Clinical Interventions in Aging

II March 2015

Number of times this article has been viewed

\author{
Istvan Gyalai-Korpos ${ }^{1,2}$ \\ Oana Ancusa ${ }^{1,2}$ \\ Tiberiu Dragomir ${ }^{1,2}$ \\ Mirela Cleopatra Tomescu, ${ }^{1,2}$ \\ losif Marincu' ${ }^{1,3}$ \\ 'University of Medicine and \\ Pharmacy, ${ }^{2}$ Cardiology Department, \\ City Hospital, ${ }^{3}$ Department of \\ Epidemiology and Infectious Diseases, \\ Victor Babes University of Medicine \\ and Pharmacy, Timisoara, Romania
}

Correspondence: Mirela Cleopatra Tomescu

2nd Eftimie Murgu Square, Timisoara

30004I, Romania

Tel +40 722979516

$\mathrm{Fax}+40220636$

Email tomescu.mirela@umft.ro
Purpose: The purpose of this prospective study was to identify factors associated with prolonged hospitalization, readmission, and death in elderly patients presenting heart failure with reduced ejection fraction.

Patients and methods: All consecutive patients aged $\geq 65$ years discharged with a diagnosis of acute new-onset heart failure and a left ventricular ejection fraction (LVEF) $\leq 45 \%$ were included and followed up for 1 year. The variables associated with outcomes were analyzed in univariate and multivariate logistic regression. For the independent predictors identified by multivariate analysis, receiver operating characteristic (ROC) analysis was performed.

Results: A total of 71 patients were included in the study. The patient mean age was 72.5 years, $50 \%$ were female, and the mean LVEF was $31.25 \% \pm 5.76 \%$. In all, 34 (48\%) patients experienced prolonged hospitalization, and this was independently associated with patients who were living in a rural area $(P=0.005)$, those with a New York Heart Association functional class of $4(P<0.001)$, the presence of comorbidities $(P=0.023)$, chronic obstructive pulmonary disease (COPD) infectious exacerbation $(P<0.001)$, and chronic kidney disease $(P=0.025)$. In the multivariate analysis, only COPD infectious exacerbation was independently associated with prolonged hospitalization $(P=0.003)$. A total 19 patients $(27 \%)$ experienced readmissions during the 1-year follow up, of which 12 (17\%) had cardiovascular causes and seven (10\%) had noncardiovascular causes. The following independent variables associated with rehospitalizations were outlined in the univariate analysis: infections $(P<0.020)$; COPD infectious exacerbation $(P=0.015)$; one or more comorbidity $(P<0.0001)$; and prolonged baseline hospitalization $(P<0.0001)$. During the multivariate analysis, it was found that the independent predictors of readmissions were the presence of comorbidities $(P<0.001)$ and prolonged baseline hospitalization $(P<0.01)$. The 1 -year mortality rate was $9.8 \%$, with no significant difference between cardiovascular (5.6\%) and noncardiovascular (4.2\%) deaths. The only independent predictive variable for mortality was a New York Heart Association NYHA functional class 4 at baseline hospitalization $(P=0.001)$.

Conclusion: Elderly patients are at high risk for prolonged hospitalization, readmission, and death following a first hospitalization for heart failure with reduced ejection fraction. The most powerful predictors for outcomes are the severity of heart failure, the presence of comorbidities, and prolonged hospitalization at baseline.

Keywords: reduced ejection fraction, outcome predictors, comorbidities, acute new onset heart failure, left ventricular ejection fraction

\section{Introduction}

Heart failure (HF) is the most common discharge diagnosis in patients older than 65 years of age; it is also a common cause of hospital readmission and death, and it presents with a significant financial burden worldwide. ${ }^{1,2}$ Although HF is primarily a disorder 
of the elderly, there is a lack of evidence-based data from randomized clinical trials pertaining to HF in this age-group. In most clinical trials, the elderly are underrepresented, often accounting for $30 \%-50 \%$ of cases. ${ }^{3}$ Despite the significant progress being made in the treatment strategies used for $\mathrm{HF}$ in adults, the prevalence of HF is increasing, and its prognosis is worsening in the elderly. ${ }^{4,5} \mathrm{~A}$ possible explanation for this fact is that most clinical trials have enrolled patients with a mean age of 60-65 years and have excluded those who had a severely impaired renal function. In the real world, however, approximately two-thirds of the elderly admitted to hospital for aggravated HF have stage 3-5 chronic kidney disease (CKD). ${ }^{6,7}$ The elderly present more frequently with comorbidities and require the use of multiple drugs for treatment; polypharmacy is thus an important problem. ${ }^{8}$ Although the association of multiple drugs often represents the recommended strategy in the treatment of HF, the simultaneous use of numerous drugs may increase the risk of drug interactions and adverse effects. It also may reduce the adherence to pharmacological therapies. ${ }^{9}$ Thus, it is possible that the treatment strategies that found to be effective in adult HF patients with reduced left ventricular ejection fraction (LVEF) are less applicable to elderly HF patients.

Accordingly, the purpose of this study was to identify the factors associated with prolonged hospitalization, readmissions, and death in elderly patients, based on variables obtained at the time of first hospital admission for HF with reduced $\mathrm{LVEF}$.

\section{Material and methods Patient selection}

The study included all patients aged $\geq 65$ years who were discharged from the Cardiology Department of the City Hospital Timisoara with a diagnosis of acute new-onset HF, from January 2013 until November 2013. A diagnosis of HF was made based on patient's symptoms, physical signs, chest radiography, and an echocardiographically determined LVEF $<45 \%$ (Simpson method), according to the European Society of Cardiology Guidelines for the diagnosis of HF. ${ }^{10,11}$ Exclusion criteria were: a diagnosis of acute myocardial infarction, acute myocarditis, acute pericarditis, and acute pulmonary thromboembolism, and the need for cardiovascular surgery.

The study was advised by the ethics committee at our hospital. Before enrollment, all patients signed an informed consent to participate in the study, in accordance with the Declaration of Helsinki. Before obtaining the consent, the patient was given sufficient time and opportunity to inquire about the details of the study; they then decided whether to participate in the study or not.

\section{Data extraction}

Baseline data were extracted from the patients' hospital records, and included the patients' age, sex, New York Heart Association (NYHA) functional class on admission, the primary cause of HF, laboratory data, chest X-ray findings, echocardiographic data, and medical history. The medical history included data regarding smoking, obesity, coronary artery disease, hypertension, valvular disease, previous myocardial infarction, atrial fibrillation, diabetes, chronic obstructive pulmonary disease (COPD), CKD, a history of stroke, malignancy, psychiatric disorders, neurologic disorders, thyroid gland disorders, anemia, osteoporosis, osteoarthritis, and infections. The types of infections that were included in the study were COPD exacerbation, pneumonia, urinary tract infections, skin infections, and infective endocarditis.

\section{Definition of covariates}

$\mathrm{HF}$ with reduced ejection fraction (HFREF) was defined as HF with an LVEF value $\leq 45 \%$. An echocardiographic evaluation was done within the first 24 hours of hospitalization, using a Vivid S5 echocardiograph. M-mode, two-dimensional, pulsed, and continuous Doppler, as well as tissue Doppler imaging of the tissues, were performed in all patients. LVEF was calculated using the Simpson method, which encompassed evaluating the end-diastolic volume and left ventricular end-systolic volume. ${ }^{10,11}$ The ischemic etiology of HF was considered in patients with a history of coronary artery disease, documented myocardial infarction, or angina. ${ }^{12}$ A patient was considered to have hypertension when high blood pressure was evident during hospitalization ( $\geq 140 / 90 \mathrm{mmHg}$ ), when there was a previous diagnosis of hypertension, or when the patient had a normal blood pressure with ongoing antihypertensive therapy. HF was considered to be the result of hypertension if the patient had a documented diagnosis of hypertension and presented with echocardiographic findings that documented significant left ventricular hypertrophy. ${ }^{13}$ Valvulopathies were identified by the patient's history, a physical examination, and the patient's echocardiographic data. ${ }^{14}$ A valvular etiology of HF was considered in the presence of moderate or severe valvular disease. A peripheral artery disease diagnosis was based on the patient's history, a physical examination, the Ankle Brachial Index results, and a Duplex ultrasound. ${ }^{15}$

CKD was diagnosed in the presence of an estimated glomerular filtration rate $<90 \mathrm{~mL} / \mathrm{min} / 1.73 \mathrm{~m}^{2} .{ }^{16}$ Diabetes 
mellitus was diagnosed according to World Health Organization (WHO)/International Diabetes Federation guidelines, based on the presence of any one of the following: a glycated hemoglobin (HbA1c) level $\geq 6.5 \%$; a fasting plasma glucose level $\geq 7.0 \mathrm{mmol} / \mathrm{L}(126 \mathrm{mg} / \mathrm{dL})$; or a plasma glucose level $\geq 11 \mathrm{mmol} / \mathrm{L}$ (200 mg/dL) 2 hours after a $75 \mathrm{~g}$ oral glucose load. ${ }^{17}$ Obesity was defined as a body mass index $\geq 30 \mathrm{~kg} / \mathrm{m}^{2} .{ }^{17}$ We used the WHO criterion for the presence of anemia in adults, which was marked as hemoglobin value $<12.5 \mathrm{~g} / \mathrm{dL} .{ }^{21} \mathrm{~A}$ diagnosis of COPD was based on the Global Initiative for Chronic Obstructive Lung Disease (GOLD) criteria. ${ }^{19}$ COPD exacerbation was defined as:

... a sustained worsening of the patient's condition, from

the stable state and beyond normal day-to-day variations,

that is acute in onset and necessitates a change in regular

medication in a patient with underlying COPD. ${ }^{20}$

Bacterial infections requiring antibiotic treatment at baseline HF hospitalization were classified as COPD exacerbation, pneumonia, urinary tract infection, skin infection, or infectious endocarditis. Other comorbidities were registered in the medical chart if present in the patient's medical history. These included: neurologic disorders, such as Parkinson's disease, cognitive impairment, and Alzheimer's disease; psychiatric disorders, such as depression and anxiety; and thyroid gland disorders, including hyper- or hypothyroidism. An osteoporosis diagnosis was based on dual energy X-ray absorptiometry (DXA), which was defined by a bone mineral density (BMD) T-score, assessed at the femoral neck, that was -2.5 or lower standard deviation (SD) units below the healthy young adult mean. ${ }^{21} \mathrm{~A}$ diagnosis of degenerative osteoarthritis was based on the patient's medical history, clinical examination, and X-ray findings.

\section{Follow up and outcomes}

Prolonged hospitalizations, hospital readmissions, and deaths were assessed as adverse outcomes. Patients with prolonged baseline hospitalizations were considered as those who exceeded the 75th percentile of hospitalization length, as measured in days. All-cause readmissions were registered during the follow-up period of 1 year. The causes of readmissions were assessed by examining the patients' hospital records. Readmissions due to worsening HF, atrial fibrillation, hypertensive crisis, acute coronary syndrome, pulmonary embolism, stroke, or acute peripheral ischemia were considered as cardiovascular readmissions. Other causes of readmissions were classified as noncardiovascular readmissions. Cardiovascular deaths were defined as deaths due to $\mathrm{HF}$, acute myocardial infarction, malignant arrhythmias (sudden death), or aortic dissection. All other deaths were classified as noncardiovascular deaths. The cause of death was ascertained from the patient's hospital records or by contacting the patient's physician by phone.

\section{Statistical analysis}

Continuous data were expressed as mean \pm SD when normally distributed; they were expressed as the median (interquartile range $[\mathrm{IQR}]$ ) when not normally distributed or skewed. Categorical data were presented as number (percent). The association between the variables and follow-up outcomes was analyzed by calculating the odds ratio and confidence intervals (CI) in the univariate analysis. The multivariate analysis included the significant independent variables that were validated in the univariate analysis. For the independent predictors that were identified by the multivariate logistical regression, receiver operating characteristic (ROC) analysis was performed. The cumulative mortality rate observed during the 1st year following the initial (baseline) hospitalization was analyzed using Kaplan-Meier curves, with the log-rank test used to compare between groups. A $P$-value $<0.05$ was considered statistically significant Statistical analysis was performed using MedCalc 12.3.0.0 statistical software for Windows.

\section{Results \\ Baseline characteristics}

All 71 consecutive elderly patients with a first hospitalization for acute new-onset HF with LVEF $<45 \%$ were included in the analysis. The patient mean age was $72.5 \pm 5.5$ (range 65-84) years, and $50 \%$ of the patients were women, and it was observed that $42 \%$ of participants were living in villages. The demographic data are presented in Table 1.

The mean duration of baseline hospitalization was $9 \pm 4$ (range 3-22) days, with a median of 8 days (IQR: 5-12 days) days. In all, 34 patients (48\%) had prolonged hospitalization, which was defined as a baseline hospitalization stay longer than 12 days ( $>75$ percentile of hospitalization length).

As presented in Table 2, the results of the univariate analysis indicated that the rural inhabitance $(P=0.005)$, NYHA functional class $4(P<0.001)$, the presence of one or more comorbidity $(P=0.023)$, an infectious exacerbation of COPD $(P<0.001)$, and the presence of $\mathrm{CKD}(P=0.025)$ were significantly associated with a hospitalization length longer than 12 days.

During the multivariate analysis, it was determined that only the infectious exacerbation of COPD was independently associated with prolonged hospitalization $(P<0.001)$. 
Table I Baseline characteristics of the 7I elderly patients newly hospitalized for HFREF

\begin{tabular}{|c|c|}
\hline Variables & Values \\
\hline Age, years (range) & $72.7 \pm 5.5(65-84)$ \\
\hline Sex, female & $35(50 \%)$ \\
\hline Rural residing & 30 (42\%) \\
\hline \multicolumn{2}{|l|}{ NYHA functional class } \\
\hline 3 & $43(61 \%)$ \\
\hline 4 & $28(39 \%)$ \\
\hline LVEF (\%) & $31.25 \pm 5.76$ \\
\hline \multicolumn{2}{|l|}{ Etiology } \\
\hline Ischemic & $30(42 \%)$ \\
\hline Hypertensive & $21(30 \%)$ \\
\hline Valvular & $20(28 \%)$ \\
\hline Old MI & $14(20 \%)$ \\
\hline Atrial fibrillation & $28(39 \%)$ \\
\hline Obesity & $23(32 \%)$ \\
\hline Present smoking & $20(28 \%)$ \\
\hline Comorbidities $\geq 1$ & $39(55 \%)$ \\
\hline COPD & $32(45 \%)$ \\
\hline Diabetes & $19(26 \%)$ \\
\hline Peripheral artery disease & $12(17 \%)$ \\
\hline Chronic kidney disease & 45 (63\%) \\
\hline Diabetes mellitus & $17(24 \%)$ \\
\hline Anemia & $14(20 \%)$ \\
\hline Bacterial infections & 40 (56\%) \\
\hline COPD infectious exacerbation & $25(35 \%)$ \\
\hline Pneumonia & $6(8.4 \%)$ \\
\hline Urinary tract infection & $4(5.6 \%)$ \\
\hline Skin infection & $4(5.6 \%)$ \\
\hline Infective endocarditis & $\mathrm{I}(\mathrm{I} .4 \%)$ \\
\hline Chronic kidney disease & $45(63 \%)$ \\
\hline Osteoporosis & $10(14 \%)$ \\
\hline Degenerative osteoarthritis & 27 (38\%) \\
\hline History of stroke & $6(8 \%)$ \\
\hline History of cancer & $3(4 \%)$ \\
\hline Neurologic disorders & $7(10 \%)$ \\
\hline Psychiatric disorders & $3(4 \%)$ \\
\hline Thyroid gland disorder & $6(8 \%)$ \\
\hline
\end{tabular}

Notes: Data are presented as mean \pm standard deviation for continuous variables and as number (\%) for continuous variables.

Abbreviations: COPD, chronic obstructive pulmonary disease; HFREF, heart failure with reduced ejection fraction; LVEF, left ventricular ejection fraction; MI, myocardial infarction; NYHA, New York Heart Association.

The ROC curve for this variable is shown in Figure 1 (area under the ROC curve $[\mathrm{AUC}]=0.789$; sensitivity $=92.9$; specificity $=64.9$ ).

A total 19 patients (27\%) experienced readmissions during the 1-year follow up, of which 12 (17\%) had cardiovascular and seven (10\%) had noncardiovascular causes. Univariate analysis revealed that the variables associated with rehospitalizations were the presence of any infection $(P<0.020)$, the infectious exacerbation of COPD $(P=0.015)$, one or more comorbidity $(P<0.0001)$, and prolonged baseline hospitalization $(P<0.0001)$. Multivariate logistic regression analyses indicated that the independent predictors of readmission were the presence of comorbidities $(P<0.001)$ and prolonged hospitalization at baseline $(P<0.01)$. As shown in Figure 2, the difference between the AUCs of the two variables was not statistically significant $(P=0.60)$.

During the 1-year follow-up period, seven deaths occurred (9.8\%), of which four (5.6\%) were assessed as having cardiovascular causes, while three $(4.2 \%)$ were noncardiovascular. The only independent predictive variable for mortality was an NYHA functional class 4 at baseline hospitalization $(P=0.001)$. The ROC curve for this variable is presented in Figure 3 (sensitivity $=85.7$; specificity $=65.6$; $\mathrm{AUC}=0.757$ ).

As shown by the Kaplan-Meier survival curves (Figure 4), there was no significant difference between the cardiovascular and noncardiovascular 1-year mortality rates among elderly HF patients with LVEF $<45 \%$ (log-rank $P=0.943)$.

\section{Discussion}

This was a prospective cohort study involving 71 patients with acute new-onset HF with LVEF $<45 \%$. Fifty percent were male, and the mean age of the participants was 72.5 (range 65-84) years. The mean age of our patients was slightly higher when compared with that of the patients from other studies, for eg, the Japanese Cardiac Registry of Heart Failure in Cardiology Report (JCARE-CRAD) ${ }^{5}$ (mean age of 71 years) and the EuroHeart Failure Survey II $^{7}$ (mean age of 70 years), but lower than that of the recently published French national observational study (mean age of 78 years). ${ }^{22}$

The median baseline hospitalization length for HF patients was 8 days (IQR: 5-12 days). A prolonged hospitalization stay ( $>12$ days) was observed for 34 patients (48\%). In the absence of a standardized definition of prolonged hospitalization, we decided to use a cutoff point of the 75th percentile of hospitalization length measured in days; this cutoff has been used in previous study as well. ${ }^{23}$ Factors associated with an index hospitalization longer than 12 days included patients living in a rural area, those with an NYHA functional class of 4 , as well as those with one or more comorbidity, CKD, and an infectious exacerbation of COPD. Multivariate analysis indicated that the only independent predictor for prolonged hospitalization was the infectious exacerbation of COPD $($ AUC $=0.789)(P<0.001)$. The close association between COPD and HF has been increasingly studied within the last 15 years. There is evidence that patients with COPD are at a significantly higher risk for congestive HF and that acute 
Table 2 Results of univariate logistic regression analysis of a prolonged hospitalization of elderly patients with HFREF ( $>12$ days versus $\leq 12$ days)

\begin{tabular}{|c|c|c|c|}
\hline Variables & Odds ratio & $95 \% \mathrm{Cl}$ & $P$-value \\
\hline Sex, female & 0.422 & $0.162-1.092$ & 0.076 \\
\hline Rural residing & 4.000 & $1.50 \mathrm{I}-10.657$ & $0.005^{*}$ \\
\hline NYHA class 4 & 0.086 & $0.021-0.347$ & $<0.001$ \\
\hline Ischemic etiology & 0.408 & $0.151-1.101$ & 0.076 \\
\hline Hypertensive etiology & 0.606 & $0.209-1.757$ & 0.356 \\
\hline Valvular etiology & 0.442 & $0.15|5-1.29|$ & 0.135 \\
\hline Old MI & 1.140 & $0.27 I-4.795$ & 0.857 \\
\hline Atrial fibrillation & 1.150 & $0.443-2.982$ & 0.773 \\
\hline Obesity & $\mathrm{I} .04 \mathrm{I}$ & $0.379-2.860$ & 0.936 \\
\hline Present smoking & 2.659 & $0.943-7.493$ & 0.064 \\
\hline Comorbidities $\geq 1$ & 4.833 & $0.02|-| 8.877$ & 0.023 \\
\hline COPD & 0.928 & $0.364-2.368$ & 0.877 \\
\hline Diabetes mellitus & 0.957 & $0.343-2.254$ & 0.937 \\
\hline Infections & 5.525 & I.583-19.272 & 0.073 \\
\hline COPD exacerbation & 10.180 & $3.006-34.482$ & $<0.00 I^{*}$ \\
\hline Pneumonia & 1.096 & $0.205-5.842$ & 0.913 \\
\hline UTI & 0.343 & $0.033-3.47 \mid$ & 0.365 \\
\hline Skin infections & 1.093 & $0.145-8.226$ & 0.930 \\
\hline Infective endocarditis & 3.358 & $0.132-85.269$ & 0.462 \\
\hline Chronic kidney disease & 6.000 & $|.248-28.84|$ & $0.025^{*}$ \\
\hline Osteoporosis & 0.414 & $0.098-1.755$ & 0.231 \\
\hline Osteoarthritis & 0.470 & $0.184-1.203$ & 0.115 \\
\hline Stroke history & 0.142 & $0.007-|.87|$ & 0.231 \\
\hline Cancer history & 0.530 & $0.045-6.127$ & 0.611 \\
\hline Neurologic disorders & 0.400 & $0.072-2.214$ & 0.294 \\
\hline Psychiatric disorders & 0.530 & $0.045-6.127$ & 0.611 \\
\hline Thyroid gland disorders & 0.193 & $0.021-1.752$ & 0.144 \\
\hline
\end{tabular}

Note: $* p<0.05$.

Abbreviations: $\mathrm{Cl}$, confidence interval; COPD, chronic obstructive pulmonary disease; HFREF, heart failure with reduced ejection fraction; MI, myocardial infarction; NYHA, New York Heart Association; UTI, urinary tract infection.

exacerbations of COPD may trigger HF aggravation, by means of a systemic inflammatory mechanism. ${ }^{24,25}$

The readmission rate of the 71 elderly HFREF was $27 \%$ during the 1-year follow-up period, with slightly more frequently occurring cardiovascular $(17 \%)$ than noncardiovascular $(10 \%)$ causes observed in this cohort $(P=0.329)$. Factors associated with the readmission of elderly HF patients included prolonged baseline hospitalizations, as well as the presence of comorbidities, infections, and COPD exacerbation. During the multivariate analysis, we found two independent predictors of hospital readmissions among the elderly included in our study: the presence of comorbidities (AUC $=0.772$ ), followed by prolonged initial hospitalization (AUC $=0.732$ ). One possible explanation for this finding is that patients that require prolonged hospitalization may have had a worse health status and an increased need for postdischarge medical care than those who did not. ${ }^{26,27}$

The 1-year all-cause mortality rate in our study was $9.8 \%$, with no significant difference between cardiovascular deaths (5.6\%) and noncardiovascular deaths (4.2\%) (logrank $P=0.943$ ). The only independent predictor for 1 -year mortality in the elderly patients with HFREF included in our study was an NYHA functional class of 4 at initial hospitalization $(\mathrm{AUC}=0.757)(P=0.001)$. The mortality rate in our study, which included elderly HF patients with a mean age of 72 years, was lower compared with other studies. For instance, Huynh et $\mathrm{al}^{28}$ found a 1 -year mortality rate of $25 \%$ in HF patients, with a mean age of 79.2 years following discharge. The Cardiovascular Health Study ${ }^{26}$ also reported high rates of death and readmissions among its cohort of elderly patients, who represented all forms of HF (normal and decreased left ventricular function). A recently published French national observational study reported a 1-year mortality rate of $29 \%$ after a first hospitalization for HF among patients with a mean age of 78 years, ${ }^{22}$ while mortality rate in $\mathrm{HF}$ patients aged $\geq 85$ years was reported to be $44 \%{ }^{29}$ We have chosen to use the age of 65 years as a cutoff for the elderly participants in our study because it coincides with the 


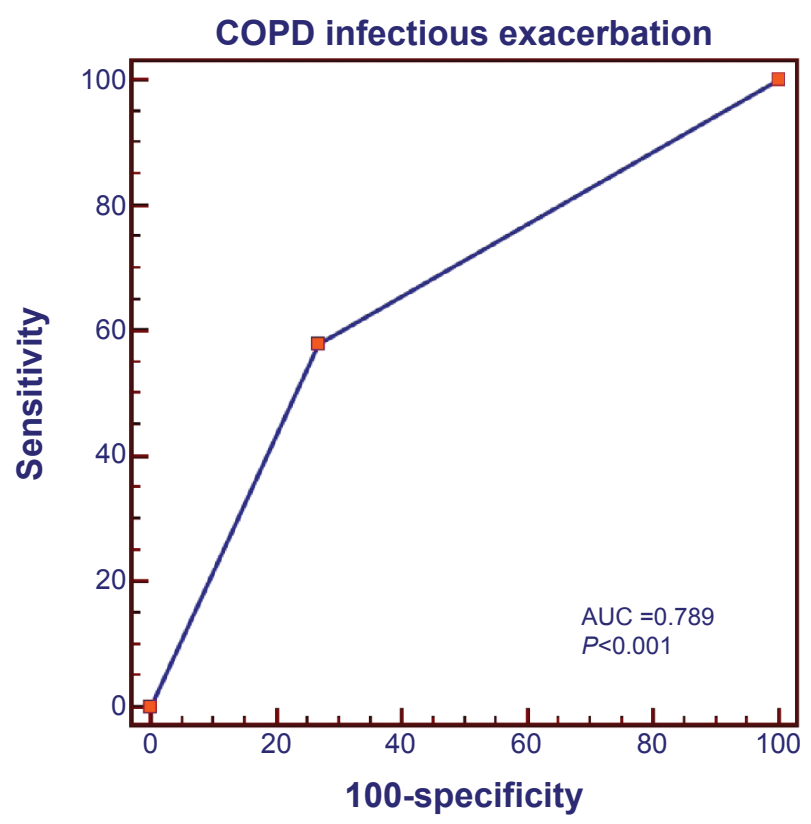

Figure I ROC curve for COPD infectious exacerbation as independent predictor for prolonged initial hospitalization in elderly patients with HFREF.

Abbreviations: AUC, area under the curve; COPD, chronic obstructive pulmonary disease; HFREF, heart failure with reduced ejection fraction; ROC, receiver operating characteristic.

retirement age in Romania and is accepted by $\mathrm{WHO}$ as the age that defines elderly individuals. ${ }^{30}$ The mean age observed for the elderly individuals in our study is also representative of Romania, where life expectancy is 74.5 years ( 71 years for men, 78 years for women), which is lower than in the central and western European countries. ${ }^{31}$

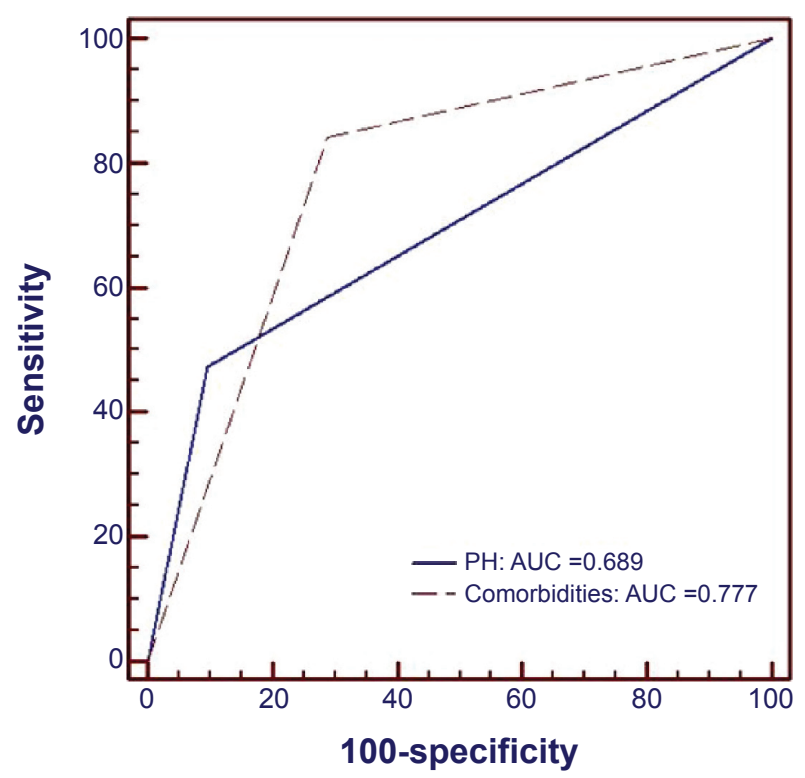

Figure 2 ROC curves for comorbidities and prolonged baseline hospitalization as independent predictors for I-year readmissions in elderly patients with HFREF. Abbreviations: AUC, area under the curve; HFREF, heart failure with reduced ejection fraction; $\mathrm{PH}$, prolonged hospitalization; ROC, receiver operating characteristic.

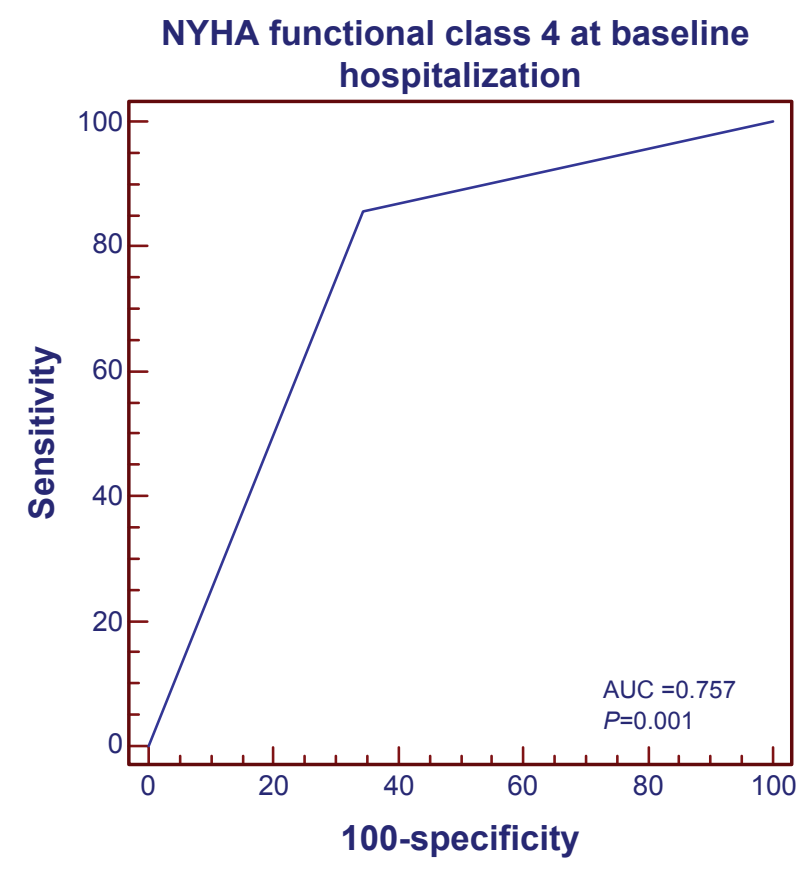

Figure 3 ROC curve for NYHA functional class 4 as independent predictor for I-year mortality in elderly patients with HFREF.

Abbreviations: AUC, area under the curve; HFREF, heart failure with reduced ejection fraction; NYHA, New York Heart Association; ROC, receiver operating characteristic.

Our study is based on data from a large, unselected patient population that represents actual clinical practice in Romania. The Cardiology Department of Timisoara City Hospital provides medical care, not only for the inhabitants of the city but also, for patients living in the villages of the western counties of Romania. The elderly patients living in villages had significantly higher rates of prolonged hospitalization $(P<0.01)$. This finding can be explained by the fact that there are no hospitals in their villages. Another possible reason is the fact that the National Health Service covers all the

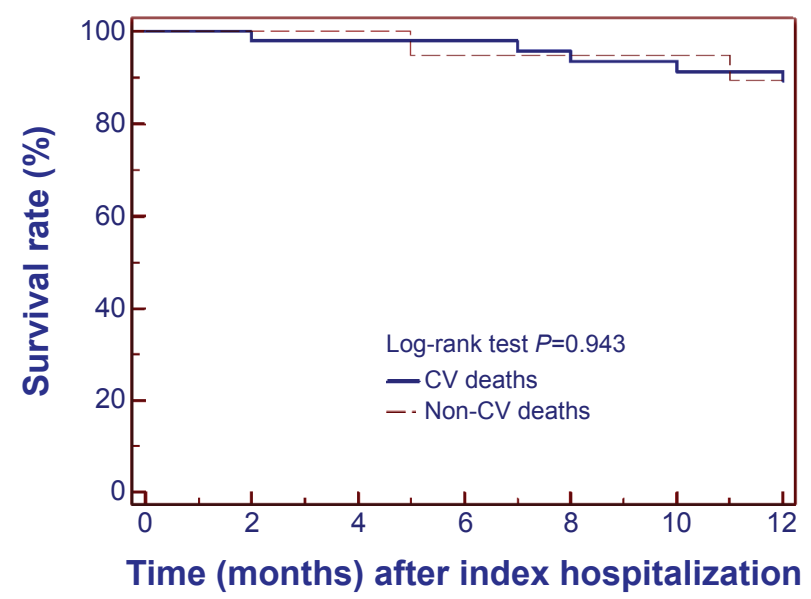

Figure 4 Kaplan-Meier event-free survival curves for cardiovascular and noncardiovascular deaths in elderly HFREF patients.

Abbreviations: HFREF, heart failure with reduced ejection fraction; CV, cardiovascular. 
hospitalization costs, while the costs of medical care services provided at the patient's home are partially covered by the patient. The elderly from the countryside often live in poorer socioeconomic conditions and prefer to be treated in hospitals. Readmission rates and mortality rates were not influenced by whether the patients resided in rural or urban areas.

\section{Study limitations}

This study has several limitations. The most important limitation is related to the small number of patients included in this study, as well as the fact that these patients were recruited from a single center. However, the advantage of this exclusively hospital-based design was that it allowed for the prospective recruitment of all patients with a first hospitalization due to HF with reduced LVEF; it also enabled their close follow up for 1 year. Although residing in a rural area was significantly associated with a prolonged initial hospitalization, we did not analyze the differences between patients who resided in rural and urban areas regarding the socioeconomic conditions (ie, income level and level of education) or the presence of comorbidities.

\section{Further developments}

Further studies with a focus on elderly people with HF are needed in order to outline the socioeconomic factors and comorbidities associated with prolonged hospitalization stay and readmission. There is also a need to develop a multidisciplinary, hospital-based strategy in order to provide adequate medical management of patients' comorbidities. A strategy to improve the ambulatory care of HF patients could reduce both the morbidity and mortality in this high-risk age group.

\section{Conclusion}

The results of this prospective, hospital-based cohort study confirm that elderly ( $\geq 65$ years) patients are at high risk for prolonged hospitalization, readmissions, and death after a first hospitalization for HFREF.

Multivariate logistic regression analyses outlined a number of independent predictors of patient outcomes. For 1-year readmissions, the infectious exacerbation of COPD, the presence of comorbidities, and a prolonged initial (baseline) hospitalization served as the predictive variables. Conversely, an NYHA functional class of 4 was predictive of 1-year mortality.

\section{Acknowledgment}

English-language editing of this manuscript was provided by Journal Prep.

\section{Author contributions}

IGK, OA, and MCT participated in the conception and design of the study, statistical analysis and interpretation of data, drafting the article, and for review of the manuscript for intellectual content. TD participated in interpretation of data, and in design and drafting of the manuscript. IM performed acquisition and interpretation of data. All authors contributed toward data analysis, drafting and revising the paper, read and approved the final manuscript, and agree to be accountable for all aspects of the work.

\section{Disclosure}

The authors reports no conflicts of interest in this work.

\section{References}

1. Linné AB, Liedholm H, Jendteg S, Israelsson B. Health care costs of heart failure: results from a randomized study of patient education. Eur J Heart Fail. 2002;2(3):291-297.

2. Redfield MM. Heart failure - an epidemic of uncertain proportions N Engl J Med. 2002;347(18):1442-1444.

3. Heiat A, Gross CP, Krumholz HM. Representation of the elderly, women, and minorities in heart failure clinical trials. Arch Intern Med. 2002;162(15):1682-1688.

4. Di Lenarda A, Scherillo M, Maggioni AP, et al; TEMISTOCLE Investigators. Current presentation and management of heart failure in cardiology and internal medicine hospital units: a tale of two worlds the TEMISTOCLE study. Am Heart J. 2003;146(4):E12.

5. Hamaguchi S, Kinugawa S, Goto D, et al; JCARE-CARD Investigators. Predictors of long-term adverse outcomes in elderly patients over 80 years hospitalized with heart failure. - A report from the Japanese Cardiac Registry of Heart Failure in Cardiology (JCARE-CARD)-. Circ J. 2011;75(10):2403-2410.

6. Komajda M, Hanon O, Hochadel M, et al. Management of octogenarians hospitalized for heart failure in Euro Heart Failure Survey I. Eur Heart J. 2007;28(11):1310-1318.

7. Komajda M, Hanon O, Hochadel M, et al. Contemporary management of octogenarians hospitalized for heart failure in Europe: Euro Heart Failure Survey II. Eur Heart J. 2009;30(4):478-486.

8. Mastromarino V, Casenghi M, Testa M. Polypharmacy in heart failure patients. Curr Heart Fail Rep. 2014;11(2):212-219.

9. McMurray JJ, Pfeffer MA. Heart failure. Lancet. 2005;365(9474): $1877-1889$.

10. Lang RM, Bierig M, Devereux RB, et al; American Society of Echocardiography's Nomenclature and Standards Committee; Task Force on Chamber Quantification; American College of Cardiology Echocardiography Committee; American Heart Association; European Association of Echocardiography, European Society of Cardiology. Recommendations for chamber quantification. Eur $J$ Echocardiogr. 2006;7(2):79-108.

11. McMurray JJ, Adamopoulos S, Anker SD, et al; ESC Committee for Practice Guidelines. ESC Guidelines for the diagnosis and treatment of acute and chronic heart failure 2012: The Task Force for the Diagnosis and Treatment of Acute and Chronic Heart Failure 2012 of the European Society of Cardiology. Developed in collaboration with the Heart Failure Association (HFA) of the ESC. Eur Heart J. 2012;33(14):1787-1847.

12. Montalescot G, Sechtem U, Achenbach S, et al; Task Force Members; ESC Committee for Practice Guidelines; Document Reviewers 2013 ESC guidelines on the management of stable coronary artery disease: the Task Force on the management of stable coronary artery disease of the European Society of Cardiology. Eur Heart J. 2013; 34(38):2949-3003. 
13. Mancia G, Fagard R, Narkiewicz K, et al. 2013 ESH/ESC guidelines for the management of arterial hypertension: the Task Force for the Management of Arterial Hypertension of the European Society of Hypertension (ESH) and of the European Society of Cardiology (ESC). Eur Heart J. 2013;34(28):2159-2219.

14. Vahanian A, Alfieri O, Andreotti F, et al; Joint Task Force on the Management of Valvular Heart Disease of the European Society of Cardiology (ESC); European Association for Cardio-Thoracic Surgery (EACTS). Guidelines on the management of valvular heart disease (version 2012). Eur Heart J. 2012;33(19):2451-2496.

15. Tendera M, Aboyans V, Bartelink ML, et al; European Stroke Organisation; ESC Committee for Practice Guidelines. ESC Guidelines on the diagnosis and treatment of peripheral artery diseases: Document covering atherosclerotic disease of extracranial carotid and vertebral, mesenteric, renal, upper and lower extremity arteries: the Task Force on the Diagnosis and Treatment of Peripheral Artery Diseases of the European Society of Cardiology (ESC). Eur Heart J. 2011;32(22):2851-2906.

16. National Kidney Foundation: K/DOQI clinical practice guidelines for chronic kidney disease: evaluation, classification, and stratification. $\mathrm{Am}$ J Kidney Dis. 2002;39(2 Suppl 1):S1-S266.

17. World Health Organization. Definition and Diagnosis of Diabetes Mellitus and Intermediate Hyperglycaemia. Geneva: World Health Organization; 2006. Available from: https://www.idf.org/webdata/docs/WHO_ IDF_definition_diagnosis_of_diabetes.pdf. Accessed February 12, 2015.

18. World health Organization Scientific Group on Nutritional Anaemias. Nutritional anaemias. Report of a WHO Scientific Group. World Health Organ Tech Rep Ser. 1968;405:1-37.

19. goldcopd.org [homepage on the Internet]. Global Strategy for Diagnosis, Management, and Prevention of Chronic Obstructive Lung Disease (GOLD). Available from: www.goldcopd.org. Accessed February 12, 2015.

20. Rodriguez-Roisin R. Toward a consensus definition for COPD exacerbations. Chest. 2000;117(5 Suppl 2):398S-401S.

21. Kanis JA, McCloskey EV, Johansson H, Cooper C, Rizzoli R, Reginster JY; Scientific Advisory Board of the European Society for Clinical and Economic Aspects of Osteoporosis and Osteoarthritis (ESCEO) and the Committee of Scientific Advisors of the International Osteoporosis Foundation (IOF). European guidance for the diagnosis and management of osteoporosis in postmenopausal women. Osteoporos Int. 2013;24(1):23-57.
22. Tuppin P, Cuerq A, de Peretti C, et al. Two-year outcome of patients after a first hospitalization for heart failure: A national observational study. Arch Cardiovasc Dis. 2014;107(3):158-168.

23. Hawkins NM, Petrie MC, Jhund PS, Chalmers GW, Dunn FG, McMurray JJ. Heart failure and chronic obstructive pulmonary disease: diagnostic pitfalls and epidemiology. Eur J Heart Fail. 2009;11(2): 130-139.

24. Wang Y, Stavem K, Dahl FA, Humerfelt S, Haugen T. Factors associated with a prolonged length of stay after acute exacerbation of chronic obstructive pulmonary disease (AECOPD). Int J Chron Obstruct Pulmon Dis. 2014;9:99-105.

25. Finkelstein J, Cha F, Scharf SM. Chronic obstructive pulmonary disease as an independent risk factor for cardiovascular morbidity. Int J Chron Obstruct Pulmon Dis. 2009;4:337-349.

26. Gottdiener JS, McClelland RL, Marshall R, et al. Outcome of congestive heart failure in elderly persons: influence of left ventricular systolic function. The Cardiovascular Health Study. Ann Intern Med. 2002; 137(8):631-639.

27. Joshi AV, D'Souza AO, Madhavan SS. Differences in hospital lengthof-stay, charges, and mortality in congestive heart failure patients. Congest Heart Fail. 2004;10(2):76-84.

28. Huynh BC, Rovner A, Rich MW. Long-term survival in elderly patients hospitalized for heart failure: 14-year follow-up from a prospective randomized trial. Arch Intern Med. 2006;166(17):1892-1898.

29. Mahjoub H, Rusinaru D, Soulière V, Durier C, Peltier M, Tribouilloy C. Long-term survival in patients older than 80 years hospitalized for heart failure. A 5-year prospective study. Eur J Heart Fail. 2008;10(1):78-84.

30. Health statistics and information systems: Definition of an older or elderly person. World Health Organization; 2015. Available from: http://www.who.int/healthinfo/survey/ageingdefnolder/en/. Accessed February 12, 2015.

31. oecd-ilibrary.org [homepage on the Internet]. OECD health statistics. Organisation for Economic Cooperation and Development (OECD); 2014. Available from: http://dx.doi.org/10.1787/health-data-en. Accessed February 12, 2015
Clinical Interventions in Aging

\section{Publish your work in this journal}

Clinical Interventions in Aging is an international, peer-reviewed journal focusing on evidence-based reports on the value or lack thereof of treatments intended to prevent or delay the onset of maladaptive correlates of aging in human beings. This journal is indexed on PubMed Central, MedLine,

\section{Dovepress}

CAS, Scopus and the Elsevier Bibliographic databases. The manuscript management system is completely online and includes a very quick and fair peer-review system, which is all easy to use. Visit http://www.dovepress. com/testimonials.php to read real quotes from published authors. 